\title{
Metodologías de Selección de Mercados Externos y Mercados Emergentes
}

Gladys Rossana Montero Santos

Montero_gr@up.edu.pe

Universidad del Pacífico. Lima. Perú

\section{Resumen}

El objetivo del presente artículo es comprobar si existe entre los académicos la preocupación por confirmar si la metodología usualmente aplicada para la selección y evaluación de mercados externos es adecuada para el caso de los llamados países emergentes. En este sentido desde el punto de vista metodológico se ha recurrido a una búsqueda ordenada de documentación respecto a dos ejes temáticos; mercados emergentes (ME) y metodologías de selección de mercados externos (SME). Los hallazgos señalan la existencia de consenso en resaltar que los mercados emergentes muestran características que los diferencian de los mercados desarrollados, situación que genera oportunidades y amenazas que deben ser consideradas para la evaluación de los mismos.

Así mismo, se han ubicado tres investigaciones que señalan las limitaciones de los métodos de selección usualmente aplicados al momento de evaluar el potencial de los mercados emergentes, proponiendo adaptaciones a las metodologías existentes. Sin embargo la comprobación empírica de estas propuestas es limitada. Finalmente consideramos importante señalar que hemos encontrado un número mayor de artículos conceptuales que estudios empíricos respecto al tema de Selección de Mercados Externos.

\section{Palabras clave:}

Mercados emergentes, economías emergentes, economías en transición, información de mercados externos, selección de mercados externos. 


\section{Introducción}

Históricamente los mercados a los cuales han aspirado las empresas inmersas en los negocios internacionales han sido aquellos pertenecientes a las economías desarrolladas. La atracción de estos mercados estaba dada por su tamaño y su constante crecimiento, definidos en función a variables macro económicas como PBI, o PBI per cápita y el incremento gradual de estos indicadores.

Sin embargo desde hace algunos años mientras los mercados desarrollados iban perdiendo dinamismo, surgió un nuevo grupo de países que fueron denominados países emergentes, economías emergentes o también llamados países en transición.

Estas economías, entre otras características presentan altas tasas de crecimiento, y lo que es más importante espacio para seguir creciendo.

Diversos estudios señalan que el futuro del crecimiento económico y del comercio mundial ira de la mano de estos países (Sunje \& Civi, 2000), (Sheth, 2011), (Cellich, 1998), (Magnusson, Westjhon, Gordon, \& Aurand, 2012), (Jansson, 2007), (Cavusgil, 1997), (Arnold \& Quelch, 1998), llegando algunas proyecciones a señalar que el resultado futuro de esta situación generará drásticos cambios en las presentes relaciones entre Estados Unidos de Norte América y Europa por una lado, y Asia y Sud América por el otro.

La Organización Mundial de Comercio señala una serie de tendencias que comprueban la creciente importancia de los mercados emergentes. Mientras que en 1980 las economías en desarrollo sólo representaban el 34\% de las exportaciones mundiales, en el 2011 su participación había aumentado al $47 \%$. Al mismo tiempo, la participación de las economías desarrolladas se redujo de $66 \%$ a $53 \%$ en el mismo período.

Estados Unidos, Japón y la Unión Europea en su conjunto, registran una participación descendiente en la cuota de las exportaciones mundiales, siendo el panorama mencionado similar en el lado de las importaciones. (World Trade Organization, 2013)

Los mercados emergentes se han convertido así en "una enorme fuente de interés, oportunidad y ansiedad durante los últimos veinte años" (Khanna \& Palepu, 2010), llamando la atención del sector académico por entenderlos y de las empresas vinculadas a los negocios internacionales por tratar de aprovechar las oportunidades que estos nuevos mercados representan.

La literatura reconoce la importancia de la correcta evaluación y selección de los mercados externos señalándola como; uno de los determinantes principales del éxito o fracaso de la internacionalización de una empresa (Papadopoulos \& Denis, 1988), decisión crítica para el éxito y supervivencia del negocio (Rahman S. H., 2000).

En función a ello diversos autores han propuesto distintos modelos de decisión para la selección de los mercados externos.

Las metodologías de selección de mercados externos fueron desarrolladas considerando condiciones y parámetros que se registraban en los países desarrollados. Por mucho tiempo la estabilidad de estos mercados dio pie a pensar que los mismos eran predecibles y bajo estos parámetros instituciones de apoyo al comercio 
internacional y académicos desarrollaron metodologías de selección de mercados externos.

La comprensión de las características del entorno de los ME es relevante, ya que permite a los investigadores evaluar la generalización de las teorías de marketing y la medida en que están limitados por el ambiente institucional de los países de altos ingresos. (Pels y Kidd, 2012).

Distintos investigadores señalan las particularidades de los mercados emergentes y de su proceso de desarrollo, más aun si los mismos son comparados con los países desarrollados.

Los autores Sunje y Civi (2000) presentan una comparación entre los mercados desarrollados y los mercados emergentes, siendo la característica más notoria en los distintos componentes del marco institucional de los ME justamente el hecho de estar en formación. (Sunje \& Civi, 2000)

Estos mercados ofrecen oportunidades pero también implican riesgos, y las metodologías de evaluación y selección de mercados externos podrían no estar considerando las características de estos mercados en su real dimensión.

Surge así el interés por conocer y analizar la aplicabilidad de estas metodologías a las economías en transición, con el fin de identificar aquella que nos permita evaluar y seleccionar nuevos mercados de destino considerando las particularidades de los mercados emergentes, teniendo como objetivo disminuir el riesgo que conlleva la diversificación de mercados. La innegable importancia de los mercados emergentes frente a la declinación de los mercados desarrollados como impulsores del crecimiento de la economía mundial hace necesario conocerlos, entenderlos y en función a ello poder considerarlos como mercados de destino dentro de una estrategia de internacionalización y de diversificación de mercados.

En función a ello, el objetivo del presente artículo es comprobar si existe entre los académicos la preocupación por confirmar si la metodología usualmente aplicada para la selección y evaluación de mercados externos es adecuada para el caso de los llamados países emergentes.

La pregunta de investigación que se propone es si los métodos de selección de mercados externos usualmente utilizados son capaces de evaluar adecuadamente el potencial de los mercados emergentes.

\section{Mercados emergentes: revisión conceptual}

\section{- Concepto y características}

El término mercados emergentes fue acuñado por los economistas de la Corporación Financiera Internacional (CFI) en 1981. (Khanna \& Palepu, 2010). Desde entonces, el término ha sido utilizado en diferentes escenarios sin que ello implique acuerdo en cuanto a la definición (Sunje \& Civi, 2000). 
De acuerdo a la Real Academia Española el término "emergente" implica un cambio ascendiente y se entiende por "transición"; a la acción y efecto de pasar de un modo de ser o estar a otro distinto.

En este sentido, resulta crucial considerar que se trata de economías que están atravesando por un proceso de transición asociado a cambios económicos. Pasar de una economía cerrada, centralizada y de baja competencia a una abierta, fragmenta y que presente un mayor nivel de competencia.

Adicionalmente debemos observar que el proceso no solo involucra cambios económicos sino que también conlleva cambios sociales y políticos (Jansson, 2007).

Estos dos últimos aspectos son lo que agregan al concepto de mercado emergente una particularidad; la posible inestabilidad del proceso de formación de su institucionalidad.

Las economías en transición deben ser capaces de mantener las políticas que vienen aplicando y de desarrollar las instituciones que apoyen el crecimiento de sus economías (Jansson, 2007).

Los autores señalan diferentes características que se encuentran presentes en los ME, las mismas que tienden a agruparse en cinco ámbitos. El primero, el ámbito regulatorio y legal. En relación a él se mencionan características como; un marco legislativo poco desarrollado o que no se cumple, débiles derechos de acreedores y accionistas, riesgo de apropiación y falta de respeto a los contratos así como poca eficiencia del sistema judicial (Pels \& Kidd, 2012).

En cuanto al ámbito socioeconómico; se mencionan como características; la variación socio económica extrema dentro de sus poblaciones, diferentes tipos de pobreza, sectores de bajos ingresos urbanos diferentes a sectores de bajos ingresos rurales, desempleo, discriminación de género y raza, violencia cotidiana, mantenimiento del status quo y comportamiento desalentador que altera la solidaridad de los grupos (Pels \& Kidd, 2012), injusticia social que involucra corrupción, delincuencia, pobreza y desnutrición, así como la presencia de reformas sociales (Jansson, 2007).

En cuanto a las características de la población, se mencionan gran tamaño de la familia, geográficamente dispersa, población joven, mano de obra joven y barata (Sunje \& Civi, 2000), (Cavusgil, 1997).

En relación al ámbito político y el rol del estado se mencionan elementos como; burocracia significativa en todo tipo de procedimientos relacionados con los negocios, diferentes niveles de corrupción, instituciones sociopolíticas con gran influencia, (Pels \& Kidd, 2012); gobernabilidad socio-política en formación (Sheth, 2011) y presencia de cambios en el sistema de gobierno del estado (Jansson, 2007).

El nivel de desarrollo de la infraestructura en estos países es descrito como deficiente o inadecuado; refiriéndose a carreteras, logística, almacenamiento, electricidad, agua corriente, gas, comunicación, información, tecnologías de transacción, redes de transferencia de datos, (Sunje \& Civi, 2000), (Pels \& Kidd, 2012), (Sheth, 2011) entre otros. 
En relación al sistema cultural, se mencionan características como; arraigo y jerarquía cultural, (Pels \& Kidd, 2012), así como alta resistencia cultural a la economía de mercado (Sunje \& Civi, 2000).

En relación a la economía y a las características de los mercados de estas economías, se señalan características como, presencia de niveles de inflación relativamente altos, mercados regidos por instituciones y no tanto por la competencia, presencia de empresas estatales y empresas con posición monopolística (Pels \& Kidd, 2012), competencia sin marca, mercado heterogéneo, (Sheth, 2011), participación del gobierno relativamente alta, marco macroeconómico, instituciones de mercado así como condiciones de mercado en proceso de desarrollo, (Sunje \& Civi, 2000), sector agrícola grande pero decreciente, (Jansson, 2007) bajo nivel de desarrollo económico, gran espacio para crecimiento futuro así como altas tasas de crecimiento, (Sunje \& Civi, 2000), (Jansson, 2007), (Arnold \& Quelch, 1998) (Cavusgil, 1997) (Sakarya, Eckman, \& Hyllegard, 2007).

Arnold y Quelch mencionan que hay tres aspectos de la economía de un país que a menudo subyacen detrás de varias definiciones en torno a los ME. El primero es el nivel absoluto del desarrollo económico, el segundo es el ritmo relativo de desarrollo económico y en tercer lugar está el sistema; si un país está en el proceso de liberalización de la economía, a veces se define como una "economía en transición" (Arnold \& Quelch, 1998)

En cuanto a la posible inestabilidad del proceso de transformación de los ME, Sunje y Civi mencionan; una fuerza motriz de todo ME es la calidad de sus reformas económicas y políticas, que es al mismo tiempo una zona altamente riesgosa (Sunje \& Civi, 2000), mientras que Jansson los describe el proceso como complejo, turbulento y volátil. (Jansson, 2007).

- Importancia de los ME

La importancia detrás de la denominación "emergente", es que ella ha sido utilizada con el fin de mostrar el cambio en el poder económico global. Investigadores e instituciones internacionales concuerdan en la importancia actual y futura de los países emergentes como motores del crecimiento global (Arnold \& Quelch, 1998), (Cavusgil, 1997), (Jansson, 2007).

Cavusgil señala que los ME son atractivos tanto para la colocación de productos como para la subcontratación, indicando adicionalmente que muchos países emergentes son una gran influencia política en su región así como motores de sus economías regionales (Cavusgil, 1997).

\section{- Importancia de la Selección De Mercados Externos (SME)}

La selección de a qué país ingresar es una decisión crítica (Douglas \& Craig, 2011). La correcta selección de los mercados externos para la expansión internacional es importante debido a i) puede ser un determinante principal del éxito o fracaso, especialmente en los primeras etapas de la internacionalización, ii) las decisiones respecto al mercado objetivo preceden el desarrollo y por lo tanto influyen en la 
naturaleza de los programas de marketing internacional, iii) La naturaleza y la ubicación geográfica de los mercados seleccionados afectan la habilidad de la empresa para coordinar sus operaciones externas. (Papadopoulos \& Denis, 1988).

La identificación y selección de mercados extranjeros atractivos es una de las preocupaciones más importantes en la exportación. Este proceso requiere el uso de información relevante, oportuna y útil (Evirgen, Bodur, \& Cavusgil, 1993).

La incorrecta selección del mercado externo puede ser costosa y colocar a la empresa en una posición estratégica desfavorable. Esta incorrecta selección genera costos de oportunidad que se traducen en una menor rentabilidad de la que hubiese sido obtenida de haberse considerado más a fondo la SME (Papadopoulos \& Denis, 1988).

\section{-Metodología de Selección de Mercados Externos}

En la evaluación de las oportunidades de los mercados internacionales para la selección de un mercado objetivo, las empresas típicamente han adoptado un planteamiento gradual y secuencial, enfocándose en primer término en información a nivel macro, para luego seleccionar un número limitado de países a ser examinados con mayor profundidad (Cavusgil, 1985), (Papadopoulos \& Denis, 1988), (Kumar, Stam, \& Joachimsthaler, 1994), (Douglas \& Craig, 2011), (Rahman S., 2000)

Estos autores también subrayan la importancia de la realización del proceso de SME de forma sistemática, lo que sucede cuando las empresas utilizan un proceso lógico y formal.

Un enfoque sistemático para la SME está asociado con el establecimiento de criterios de selección, un filtrado preliminar de mercados potenciales, la estimación del potencial de ventas de la industria en cada mercado y la estimación de las ventas de la empresa en cada uno de los mercados bajo estudio.

Existe un consenso en los pasos que debe incluir un proceso de Selección de Mercados Externos (SME); ellos son Filtración (revisión preliminar), Identificación (en profundidad) y Selección (final); en contraposición existe un consenso limitado respecto a qué criterios utilizar para estimar el potencial de un mercado, la importancia relativa de cada criterio y como aplicarlos.

\section{GRÁFICO N 1: PASOS EN EL PROCESO DE SELECCIÓN DE MERCADOS EXTERNOS}

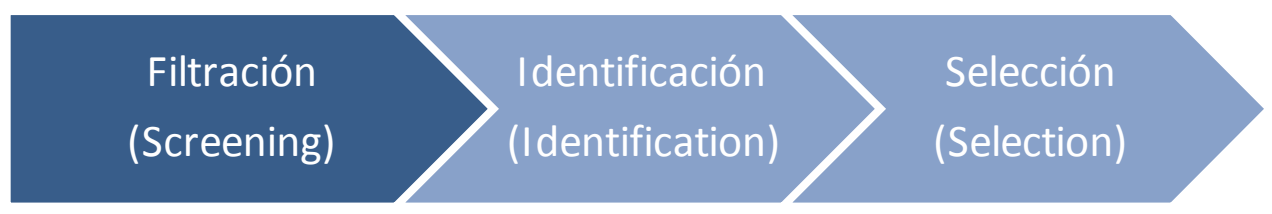

Elaboración Propia 
Los autores Berbel, Ramón y Vasquez señalan que la valoración o revisión preliminar identifica mercados potenciales como candidatos para un siguiente análisis en mayor profundidad, y que en esta etapa se emplean indicadores a nivel macro para eliminar aquellos mercados que no se ajustan a los objetivos de la empresa (por ejemplo, tamaño de mercado, tasa de crecimiento, grado de ajuste entre las preferencias del consumidor y el producto, rivalidad competitiva, etc.) En la etapa de identificación se valora el atractivo de la industria y se estiman costes e ingresos de una lista de países más reducida como consecuencia de la etapa previa. Por último, la etapa de decisión final determina el mercado que mejor se ajusta a los objetivos de la empresa y a los recursos disponibles. (Berbel Pineda, Ramón Jerónimo, \& Vásquez Carrasco, 2012).

Russow y Okoroafo revisan la literatura en torno al "filtrado" de países y encuentran que distintos autores plantean cuestiones críticas como; ¿cuáles son los criterios de selección apropiados? ¿Cómo medir y ponderar dichos criterios?, ¿están correlacionados estos criterios? Los autores añaden a esta discusión, interrogantes respecto al momento en que debe procederse al filtrado dentro del proceso de SME, la importancia relativa de cada criterio y la manera como los mismos deben emplearse. (Russow \& Okoroafo, 1996)

Rahman se centra en identificar los criterios del entorno macro económico para la SME, confirmando la importancia de la utilización de las variables tamaño de mercado y desarrollo económico para la identificación de oportunidades potenciales. (Rahman S., 2000).

Una clasificación de los diferentes planteamientos teóricos en relación a la SME es presentada por los autores Nicolas Papadopoulos y Jean - Emile Denis "Inventory, Taxonomy and Assessment of Methods for International Market Selection". (Papadopoulos \& Denis, 1988). En este documento se presenta una primera gran separación de enfoques cualitativos y cuantitativos, así como un análisis de las ventajas y limitaciones de cada enfoque.

Una primera división se refiere a los enfoques cualitativos y los cuantitativos.

\section{-Enfoques Cualitativos de SME}

Establecen objetivos y restricciones que posteriormente son considerados en el análisis. Algunos estudios se focalizan en la naturaleza, oportunidad y las fuentes de información cualitativas que pueden ser utilizadas como insumos para el proceso de SME.

El enfoque cualitativo está siendo dejado de lado, mencionándose los siguientes factores como base para esta decisión:

- $\quad$ El Sesgo Profesional, que se refleja en la selección de las variables a considerar para la SME. Los investigadores eligen variables relacionadas con su campo de conocimiento, dejando de lado otras variables relacionadas a otros campos que también deberían ser consideradas. Si bien el planteamiento cualitativo es sistemático, el mismo está abierto a opiniones potencialmente sesgadas de aquellos que proveen información y asesoramiento; y al juicio subjetivo del tomador de decisiones. (Papadopoulos \& Denis, 1988). 
- La Realidad Objetiva y Percibida: La realidad objetiva se logra a través de agrupar países en función de variables socio-económicas secundarias, mientras que la realidad subjetiva de deriva del hecho de consultar a expertos internacionales.

- Inexactitud: La inexactitud puede ser resultado de los puntos anteriormente mencionados como también surge de la imposibilidad de evaluar a un número elevado de países de manera simultánea.

Los enfoques cualitativos están limitados por su propia naturaleza al solo poder considerar un número limitado de países; y siguen estando abiertos a opiniones potencialmente sesgadas de quienes brindan información y asesoramiento y al juicio subjetivo de quien toma las decisiones. (Papadopoulos \& Denis, 1988).

\section{-Enfoques Cuantitativos de SME}

Utilizan análisis estadísticos más formales que permiten la comparación de un mayor número de mercados. Los planteamientos cuantitativos son subdivididos en dos ramas; la primera agrupa metodologías que tienen como objetivo la "agrupación de mercados" en base a semejanzas, mientras la segunda rama agrupa metodologías que tienen como objetivo la "estimación de los mercados", reuniendo metodologías que apuntan a priorizar los mercados sobre la base de su potencial.

- Metodologías de Agrupación de Mercados: Se basan en la evaluación de una amplia variedad de indicadores políticos, económicos y sociales que permiten comparar un gran número de países buscando definir agrupaciones en base a ciertas características o condiciones compartidas.

Dentro de esta categoría se encuentran modelos de "macro segmentación" que tratan de agrupar mercados en base a su situación general y no hacen ningún intento por medir los niveles de demanda (Papadopoulos \& Denis, 1988).

A nivel metodológico inicialmente estos estudios utilizaron el análisis de clúster para la identificación de países con el mismo nivel de desarrollo industrial, perfeccionándose gradualmente a través del uso de técnicas estadísticas más sofisticadas como el análisis factorial, pasando de un gran número de variables bajo estudio a grupos significativos de variables para la agrupación de los países, reforzándose la validez predictiva de los modelos desarrollados y la utilización de variables distintas al desarrollo industrial para la identificación de los grupos de países (Papadopoulos \& Denis, 1988).

La principal limitación señalada en torno a esta metodología es que el proceso de agrupación se basa exclusivamente en indicadores generales (macro) de los países que si bien pueden caracterizar adecuadamente el entorno de marketing global, carecen de especificidad en relación al producto. Así también se menciona la posibilidad de que los indicadores seleccionados sean redundantes al presentar colinealidad (Papadopoulos \& Denis, 1988)

En cuanto a los modelos de "micro segmentación" dentro de esta categoría, se encuentran estudios que han aplicado indicadores relacionados específicamente con el producto para el cual se busca identificar mercados externos. Estos estudios arrojan 
resultados que difieren dramáticamente de los resultados que arrojan los estudios realizados solo evaluando variables macro. La limitación para la aplicación de esta metodología está en la disponibilidad de datos secundarios, lo que dificulta la comparación entre países (Papadopoulos \& Denis, 1988).

Finalmente dentro de las Metodologías de Agrupación de Mercados también se presentan estudios que han optado por un método secuencial, utilizando primero una macro segmentación para luego pasar a un proceso de micro segmentación. (Papadopoulos \& Denis, 1988)

Papadopoulos y Denis también recalcan que estos métodos se basan en el postulado de que los mercados más atractivos para una empresa son los que más se parecen a los mercados en los que ya ha penetrado, lo que implica suponer que la empresa ya ha tenido éxito ingresando a un mercado externo (Papadopoulos \& Denis, 1988); o lo que es lo mismo que la empresa no está realizando una SME por primera vez, con el fin de elegir un destino para iniciar su proceso de internacionalización.

- Metodologías de Estimación de Mercados: Estas metodologías buscan evaluar los mercados externos en base a uno o más criterios y aquellos países que logran el mayor puntaje son seleccionados. Este grupo se sub divide en aquellos que se focalizan en la "Demanda Potencial Total" de los mercados externos y aquellos que se enfocan en la "Demanda Potencial de las Importaciones", es decir en el componente importado del mercado potencial.

En cuanto a los estudios que se focalizan en la Demanda Potencial Total, incluyen métodos en base a índices de factores múltiples y métodos econométricos.

En cuanto a los trabajos fundados en la construcción de índices en base a múltiples factores, los índices se derivan mayormente de dos sets de criterios; desarrollo económico y estabilidad interna y cohesión.

Los índices de factores múltiples son entendidos como medidas indirectas del potencial de mercado que se calculan mediante la asignación de valores relativos a indicadores representativos que han sido seleccionados por la intuición, el juicio experimentado, o el análisis estadístico. (Papadopoulos \& Denis, 1988).

Ente las limitaciones de este método se mencionan el uso de factores generales (macro) frente a la no aplicación de factores específicamente relacionados al producto, así como la posibilidad de que los indicadores seleccionados sean redundantes al presentar colinealidad (Papadopoulos \& Denis, 1988).

En relación a los modelos econométricos para la predicción de la demanda la metodología busca identificar variables independientes que muestren un poder de predicción sustancial a través del análisis de regresión.

La limitación de esta metodología está en la disponibilidad de data que no siempre está disponible con facilidad, especialmente en países de menor desarrollo relativo. (Papadopoulos \& Denis, 1988)

En cuanto a los estudios que se focalizan en la Demanda Potencial de las Importaciones, estos enfoques evalúan específicamente el componente importado de la 
"Demanda Aparente" de un producto dado. Siendo la demanda aparente el resultado de agregar a la producción interna de un país para un determinado producto, las importaciones y restar las exportaciones del mismo producto.

Dentro de esta clasificación se presentan tres variantes. La primera de ellas se basa en el uso de Criterios Múltiples y fue desarrollada por el Centro de Comercio Internacional. En base a esta metodología se analizan los mercados en función a sus importaciones, el crecimiento de las mismas, la cobertura de mercado (importaciones/ exportaciones) y competencia. Tiene la ventaja de ser sencilla en su aplicación, pero presenta el problema de ofrecer un bajo grado de especificidad en función a utilizar el Sistema Armonizado, sistema de clasificación internacional de mercancías que no ofrece información precisa del potencial de mercado a nivel de productos individuales. Otra limitación importante es el atraso en la publicación de las estadísticas que usualmente bordea los dos años.

La segunda metodología se focaliza en planteamientos econométricos. El método consiste en la recopilación de datos relacionados al producto bajo estudio, donde también se incluyen las estadísticas de comercio internacional, identificar las variables pertinentes y desarrollar el modelo para la estimación de la demanda.

La tercera metodología se basa en el estudio en el cambio en la participación (Shift Share Approach). Se centra en identificar cambios relativos en la participación internacional de las importaciones de varios países. El análisis calcula la tasa de crecimiento promedio de las importaciones para una "canasta" de países y compara el crecimiento de cada país contra el promedio. La diferencia entre ambos, denominado "cambio neto" (net shift), identifica mercados crecientes o en declive dentro del grupo de países bajo análisis.

Este procedimiento tiene la ventaja de considerar tanto el tamaño de las importaciones de un país como su tasa de crecimiento relativo. Por otra parte, está limitado a esos dos criterios, y por lo tanto no examina el conjunto de dimensiones estratégicas o del entorno en la SME. (Papadopoulos \& Denis, 1988)

Antes de pasar al siguiente punto de estudio, consideramos relevante mencionar la posición de un número importante de investigadores que señalan las limitaciones de los enfoques que solo contemplan el análisis a nivel macro; Douglas y Craig señalan; el elemento clave es que dos países pueden tener contextos macro-ambientales muy similares, pero pueden diferir radicalmente en sus contextos a otros niveles (Douglas \& Craig, 2011).

Al hablar de los factores macro ambientales señalan, mientras cada uno de estos diferentes factores puede ser claramente identificado, es importante recordar que interactúan entre ellos, así como también acondicionan variables a otros niveles del contexto.

Ellos recomiendan para la evaluación de oportunidades internaciones de mercado, ir a un análisis a cuatro niveles; macro, meso, micro y factores situacionales que influyen en el comportamiento de compra y consumo en relación a un producto. (Douglas \& Craig, 2011). 
Rahman recomienda que las empresas evalúen una serie de indicadores significativos para el producto bajo estudio. Estos indicadores variarán de una empresa a otra y de un producto a otro. Sosteniendo que es insuficiente para evaluar los mercados, apoyarse sólo en el PIB y la población total de cada país sin tener en cuenta cifras demográficas relevantes. Tales cifras demográficas varían en importancia según el producto (Rahman S., 2000).

\section{-Criterios, Indicadores y Ponderaciones}

Si bien hemos mencionado que no existe acuerdo en cuanto a los indicadores y ponderaciones que deben ser aplicados en cada fase del proceso de SME, presentamos en esta sección dos estudios desarrollados en base a esta problemática.

Utilizando entrevistas a profundidad en base a una muestra de exportadores turcos líderes, los autores Evirgen, Bodur y Cavusgil identifican las preferencias de información de los exportadores en la evaluación y selección de mercados externos. Estos autores identificaron diversos indicadores para cada una de las etapas del proceso de evaluación/selección de mercados.

En la primera etapa, denominada valoración preliminar, los indicadores se refieren al entorno demográfico, político, económico y socio - cultural. En la segunda etapa, donde se busca evaluar el potencial de mercado de la industria; los indicadores se refieren a las condiciones de entrada que presenta el mercado, al entorno legal, a la demanda y sus características comportamentales así como a la distribución. Finalmente, en la tercera etapa; en donde el objetivo es evaluar el potencial de las ventas, se analiza la competencia, la estructura financiera, la publicidad, la promoción y la comunicación entre otros factores. (Evirgen, Bodur, \& Cavusgil, 1993)

Los resultados ponen de manifiesto que existen diferencias significativas en la importancia percibida de diferentes tipos de información a través de las industrias y los principales destinos de exportación, la información sobre la estructura financiera del país de destino, así como sobre la competencia y condiciones de entrada resultan ser valoradas como las más importantes. (Evirgen, Bodur, \& Cavusgil, 1993).

Wood y Robertson (2000) realizan una extensa revisión de la literatura relacionada con la información que puede ser útil al momento de evaluar mercados internacionales. Identifican un total de doscientos indicadores, los cuales reducen (para conseguir mayor operatividad) a través de un proceso de entrevistas y grupos de enfoque con representantes de agencias gubernamentales relacionadas con la exportación, de instituciones bancarias internacionales y de negocios privados con experiencia en exportación. De este proceso obtienen seis dimensiones, en donde se agrupan diferentes indicadores, presentando un ranking tanto de las dimensiones como de los indicadores considerados como los más importantes en la SME. (Berbel Pineda, Ramón Jerónimo, \& Vásquez Carrasco, 2012).

El potencial de mercado y la información legal son las dos dimensiones consideradas en primer término, la tercera dimensión es la política, la cuarta la infraestructura, la 
quinta la dimensión económica, mientras que en último lugar se encuentra la cultura. Los autores encuentran que este ranking no varía ni en función al mercado de destino (a excepción del caso de China, en donde la información relacionada a la política ocupa el segundo lugar en importancia y la relacionada al marco legal ocupa el tercer lugar) ni en función al tipo de negocio (sean productos de consumo, industriales o servicios) (Wood \& Robertson, 2000).

Los autores aclaran que encontrar a la dimensión cultural en último lugar, no significa que la cultura no sea importante; sino que resulta relevante solo después de que otros factores en torno a otras dimensiones hayan sido evaluados positivamente.

Un punto interesante mencionado por Wood y Robertson son las excepciones que encuentran en relación al ranking presentado. Ellos mencionan que en los mercados desarrollados la información relacionada a la infraestructura ocupa el quinto lugar de importancia, mientas que la información relacionada con la economía ocupa el cuarto lugar. En el caso de los mercados en vías de desarrollo (Latinoamérica, Rusia, Europa del Este, China y otras áreas de Asia y África) este orden se invierte, siendo planteados los temas logísticos antes que los económicos. (Wood \& Robertson, 2000).

- Propuestas de Adaptación de Metodologías de SME con el fin de considerar las Características de los ME

Cavusgil desarrolla una metodología para cuantificar y clasificar el potencial de mercado de 25 países identificados como países emergentes por la revista Economist.

En primer término selecciona trece variables; económicas, políticas y sociales que son agrupadas en siete "dimensiones fundamentales" a ser consideradas para determinar el atractivo general de un mercado.

A continuación desarrolla un índice estandarizando los datos referentes a las trece variables y situándolos en una escala del 1 al 100. Como tercer paso a través de entrevistas a un número selecto de profesionales y profesores de negocios internacionales (proceso Delphi) asigna una importancia relativa a cada dimensión, para finalmente combinando las siete dimensiones presentar el Índice Integral de Oportunidades de Mercado (OMOI).

Las siete dimensiones propuestas son; (1) Tamaño de Mercado, evaluado a través de la población total del país y asignándole un peso de 4/20. (2) Tasa de crecimiento del mercado, evaluada a través de la tasa de promedio anual de crecimiento de la industria, asignándole un peso de 3/20. (3) Intensidad de Mercado, calculada evaluando el gasto de las empresas y de los hogares a través de la estimación del PBI per cápita y el gasto de consumo privado per cápita en dólares, asignándole un peso de 3/20. (4) Capacidad de Consumo del Mercado, evaluada por medio del tamaño de la clase media; que para el autor es un indicador de la propagación de la base de consumo. A esta dimensión se le asigna un peso de 2/20. (5) Infraestructura Comercial, que persigue evaluar la facilidad de acceso a los canales de distribución y comunicación. Las variables utilizadas para definir esta dimensión fueron líneas telefónicas por habitante, densidad de 
carreteras pavimentadas, camiones y ómnibus per cápita, habitantes por punto de venta y finalmente cantidad de hogares con televisores a color. A esta dimensión se le asigna un peso de 2/20. (6) Libertad Económica, utiliza el índice de libertad Económica desarrollado por la Heritage Foundation (http://www.heritage.org/), incorporando de esta manera conceptos como política comercial y tributaria, política monetaria y bancaria, flujos de capital e inversión extranjera entre otros. A esta dimensión se le asigna un peso de 2/20. (7) Receptividad del Mercado; magnitud en la que un mercado externo está abierto a las importaciones de los Estados Unidos, la cual estima en función a las importaciones per cápita desde los Estados Unidos y el promedio anual de crecimiento de las importaciones procedentes de los Estados Unidos en los últimos cinco años; asignándole a esta dimensión un peso de 4/20.

El autor reconoce limitaciones del OMOI, indicando que el índice arroja una medición agregada del atractivo de los mercados emergentes, acotando su utilidad a la etapa inicial de evaluación y clasificación de estos países, recomendando un análisis más exhaustivo al enfrentarse una empresa a la decisión de ingreso y establecimiento en un mercado.

Así mismo señala que esta metodología es más adecuada para empresas exportadoras, y remarca la importancia de la confiabilidad de la información estadística (Cavusgil 1997).

Evaluando las siete dimensiones notamos que el autor evalúa las oportunidades que presentan los ME más que los riesgos que conllevan, aunque menciona que el riesgo está inmerso en la dimensión de receptividad de mercado debido a que la misma refleja el grado de apertura del país para con las importaciones de origen norte americano (Cavusgil, 1997).

Un segundo aporte es realizado por Arnold \& Quelch; estos autores tratan de cuatro áreas en las que sostienen las empresas deben repensar sus modelos de marketing al dirigirse a países emergentes; i) el momento del ingreso, ii) la evaluación del mercado, iii) la política respecto al producto y iv) la política respecto a los socios (Arnold \& Quelch, 1998). En este acápite nos centraremos exclusivamente en el segundo punto, los aportes de los autores respecto a la evaluación de los mercados de países emergentes.

Señalan los motivos por los que el método tradicional de evaluación de mercados no es aplicable a los mercados emergentes; i) con frecuencia tales modelos dependen de data macroeconómica y poblacional que son inexactas y desactualizadas en los países emergentes, ii) los modelos asumen la disponibilidad de datos de operaciones como niveles de ventas o número de distribuidores que en ocasiones no están disponibles y iii) los modelos son generalmente evaluaciones "instantáneas", estáticas en lugar de evaluaciones dinámicas ignorando de esta manera el potencial a largo plazo de los MEs y su rápido ritmo de cambio. (Arnold \& Quelch, 1998)

Los modelos tradicionales consideran el riesgo macroeconómico y político en la fase inicial del proceso de evaluación, lo que trae como resultado que muchos MEs de alto potencial sean descartados en la primera parte del proceso. En función a ello su aporte es "presentar un modelo guiado por la demanda del mercado considerando un ajuste por riesgo, en lugar de ajustar la demanda potencial guiado por el riesgo". (Arnold \& Quelch, 1998) 
Los autores indican que su propuesta; "Modelo Anidado Para La Evaluación De Mercados Emergentes" cuenta con tres características distintivas; i) permite un proceso de filtros sucesivos, con fases secuenciales incrementalmente discriminantes para la evaluación de mercados. Después de cada fase algunos candidatos (mercado - país) pueden ser eliminados. Esta es la base para una clasificación de los mercados (países), ii) Se basa en datos progresivamente más específicos y detallados por mercado. Parte de información demográfica y económica con el fin de evaluar la demanda futura y avanza progresivamente con datos específicos tanto de producto - mercado como país - mercado. iii) el modelo presenta consideraciones respecto al potencial de mercado a largo plazo antes que indicadores más inmediatos de riesgo país y de la "conversión del beneficio potencial".

El segundo aporte de los autores se da en relación a la Evaluación del Potencial de Mercado a Largo Plazo utilizando datos y tendencias de población y PBI. El potencial de mercado lo obtienen relacionando la población del país emergente con la diferencia entre la riqueza del mercado emergente y los mercados desarrollados.

Reconocemos un último aporte en el concepto "conversión de beneficio potencial". Los autores indican que las empresas deben ser capaces de extraer valor del mercado y que ello dependerá del riesgo político y económico y del desarrollo de condiciones adecuadas en el sector comercial como por ejemplo la infraestructura en transporte, distribución y telecomunicaciones. Este punto es interesante ya que una de las características de los mercados emergentes es el atraso en la infraestructura y pocos autores revisados respecto al tema de Selección de Mercados consideran esta dimensión en su análisis.

Sakarya, Eckman y Hyllegard (2007) también reconocen la limitación de los enfoques tradicionales al depender los mismos de criterios macroeconómicos y políticos sin tener en cuenta el dinamismo y el potencial futuro de un mercado emergente, (Sakarya, Eckman, \& Hyllegard, 2007).

El principal aporte en la investigación es el desarrollo de un enfoque especializado para la evaluación preliminar de MEs, añadiendo cuatro criterios; i) el potencial futuro de los MEs, ii) la distancia cultural entre los MEs y el país de origen, iii) la capacidad competitiva de la industria específica en los MEs y iv) receptividad del cliente a los productos de las industria extranjera y el país de origen. (Sakarya, Eckman, \& Hyllegard, 2007).

Recogen los aportes de otros autores, en el caso del potencial futuro del mercado recurren al aporte de Arnold \& Quelch (Arnold \& Quelch, 1998); en el caso de la distancia cultural recurren al índice desarrollado por Morosini et al. (1998) que a su vez se basa en los aportes de Hofstede en cuanto a distancia cultural; en el caso de capacidad competitiva recurren a Porter (1990). Señalan que el análisis del diamante nacional para la industria en evaluación ayuda a superar algunas limitaciones del análisis tradicional de Investigación de Mercados Externos, el cual asume que los países son homogéneos y el nivel de desarrollo de sus industrias se refleja en conjunto, en los indicadores generales del país, en cuanto al criterio relacionado con la receptividad del cliente. La opinión de los clientes del país anfitrión en relación al impacto de la actividad empresarial extranjera en el desarrollo económico y social, la aceptación de sus productos y marcas, así como la percepción de sus ofertas con respecto a otras 
empresas extranjeras y locales en la misma industria (competidores) se utiliza como medidas de este criterio.

Las autoras no indican de manera explícita si el enfoque de Selección de Mercados Externos que plantean es más adecuado para un modo de entrada en específico. En su bibliografía encontramos tanto documentos relacionados a las operaciones de las multinacionales como a exportaciones.

Un aporte a mencionar es que las autoras han aplicado el modelo planteado evaluando las oportunidades de la industria de textiles y confecciones de Estados Unidos en Turquía. Es decir la aplicación del modelo se da a una industria de manera específica. 


\section{Metodología}

El presente ensayo es el primer paso de un proceso de investigación que tiene como objetivo contribuir al mejor análisis de los países emergentes con el fin de evaluarlos como posibles mercados de destino en el ámbito de los negocios internacionales. En este sentido la revisión de la literatura realizada busca ser la base para un segundo objetivo, a saber la justificación para futuras investigaciones en torno al tema.

Se ha optado por una revisión de literatura narrativa o también denominada tradicional con el fin de revisar y resumir un cuerpo de literatura y formular conclusiones del tema de estudio. Esta decisión es tomada bajo el entendido que la revisión de literatura narrativa busca inspirar ideas de investigación mediante la identificación de gaps o inconsistencias en un conjunto de conocimientos, lo que permite la determinación o definición de preguntas de investigación o hipótesis futuras (Cronin, Ryan, \& Coughlan, 2008).

Desde el punto de vista metodológico se ha recurrido a una búsqueda ordenada de documentación respecto a dos ejes temáticos; mercados emergentes y metodologías de selección de mercados externos (SME). Considerando la relación del proceso de selección de los mercados externos con el éxito o fracaso de los procesos de internacionalización de las empresas, se priorizo la búsqueda y utilización de fuentes que ofrecieran tanto una perspectiva desde los negocios internacionales como desde el marketing internacional.

La literatura revisada incluye tanto investigaciones estrictamente académicas (journals) como no académicas. Esta decisión encuentra sustento en el hecho que el tema de investigación interesa tanto a profesionales del mundo académico como a profesionales del mundo de la práctica.

\section{Resultados}

En relación a los mercados emergentes la revisión bibliográfica realizada permite observar que aún no existe acuerdo en torno a su definición, mientras que sí se presenta un mayor acuerdo en torno a señalar sus características, así como el hecho de que dichas características diferencian a los mercados emergentes de los mercados desarrollados.

Si bien el ámbito económico, y los cambios que en sus economías están llevando a cabo estos mercados interesan a muchos investigadores, los cambios que se observan en los mercados emergentes abarcan otros ámbitos además del económico.

En el cuadro presentado a continuación se exponen los ámbitos en donde se ubican las principales características mencionadas por los autores en relación a los países emergentes. 


\section{CUADRO No 1: ÁMBITOS EN DONDE SE UBICAN LAS PRINCIPALES CARACTERÍSTICAS DE LOS MERCADOS EMERGENTES}

\begin{tabular}{|c|c|c|c|c|}
\cline { 2 - 5 } \multicolumn{1}{c|}{} & $\begin{array}{c}\text { Jacqueline } \\
\text { Pels y Tomas } \\
\text { Kidd }\end{array}$ & $\begin{array}{c}\text { Aziz Sunje y } \\
\text { Emin Civi }\end{array}$ & $\begin{array}{c}\text { Jagdish N. } \\
\text { Sheth }\end{array}$ & Hans Jansson \\
\hline Sistema de Regulación & $\mathrm{X}$ & $\mathrm{X}$ & $\mathrm{X}$ & $\mathrm{X}$ \\
\hline Sistema Socioeconómico & $\mathrm{X}$ & $\mathrm{X}$ & $\mathrm{X}$ & $\mathrm{X}$ \\
\hline $\begin{array}{c}\text { Gobernabilidad } \\
\text { socio-política }\end{array}$ & $\mathrm{X}$ & $\mathrm{X}$ & $\mathrm{X}$ & $\mathrm{X}$ \\
\hline Infraestructura & $\mathrm{X}$ & $\mathrm{X}$ & $\mathrm{X}$ & \\
\hline Sistema Cultural & $\mathrm{X}$ & $\mathrm{X}$ & & \\
\hline
\end{tabular}

En cuanto a las condiciones o características que diferencian a los mercados emergentes de los mercados desarrollados, se mencionan tres factores. El primero de ellos es la formación de su institucionalidad. Esta característica afecta a todos los ámbitos mencionados lo que a su vez le agrega al concepto de mercado emergente una particularidad; la posible inestabilidad del proceso de formación de su institucionalidad; ya que se entiende que las economías emergentes deben ser capaces de mantener las políticas que vienen aplicando y de desarrollar las instituciones que apoyen el crecimiento de sus economías.

El segundo factor mencionado por los autores es el de heterogeneidad, aunque en este caso esta característica no necesariamente conlleva el mismo significado. Algunos autores mencionan el término heterogeneidad con el fin de referirse a la diversa naturaleza de los países que son ahora llamados emergentes, es decir se incide en la heterogeneidad del grupo.

El caso más claro en ese sentido, es el de los países que antes formaban parte de los bloques socialistas y que en su momento iniciaron un proceso de cambios buscando pasar de una economía centralizada a una economía de libre mercado. Junto con estos países, debemos considerar como emergentes, a países latinoamericanos, africanos y asiáticos que por distintos motivos presentaban economías con un menor desarrollo relativo aunque en ellas que existiera una economía de libre mercado. En ese sentido considerar ambos grupos de países bajo la identidad de emergentes, explica la heterogeneidad del grupo.

Otro factor que le añade heterogeneidad al grupo de países llamados emergentes, es la diversidad geográfica de los mismos; como se ha mencionado dentro de los países emergentes, existen países asiáticos, latinoamericanos y africanos. Los autores presentan esta diversidad geográfica como un factor que aporta heterogeneidad al grupo. 
Desde otra perspectiva, algunos autores se refieren el término heterogeneidad incidiendo en las diferencias que internamente muestran las economías emergentes. Aquí se mencionan factores como las diferencias entre el campo y la ciudad, las diferencias en el grado de industrialización, las diferencias a nivel intra e inter industrial, así como de desarrollo e infraestructura de ciertas zonas de dichos países, frente al resto de su territorio.

En cuanto al tercer factor, sí encontramos acuerdo entre los investigadores respecto al reconocimiento del potencial de crecimiento de estos países. Los investigadores concuerdan en que el futuro de la economía mundial girará en torno a estos países; aunque algunos autores centran su interés en visualizar estos mercados principalmente como fuentes de demanda, mientras que otros añaden a esta característica la consideración de estos países para temas de distribución y relocalización de la producción a nivel mundial.

En relación al segundo tema que aborda este ensayo, las metodologías de selección de mercados externos; se presenta acuerdo en cuanto a su importancia. La SME es reconocida como factor clave para llevar a cabo un adecuado proceso de internacionalización. Así también existe consenso en torno a la necesidad de considerar un enfoque sistemático y que se trata de un proceso gradual que involucra tres pasos; filtración, identificación y selección.

\section{GRÁFICO № 1: PASOS EN EL PROCESO DE SELECCIÓN DE MERCADOS EXTERNOS}

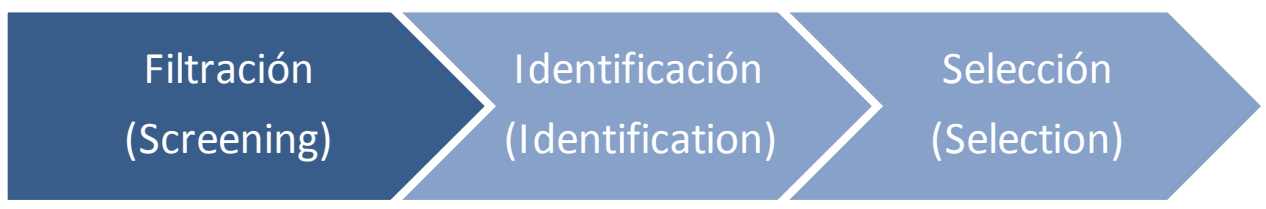

Elaboración Propia

En contraposición, existe un consenso limitado respecto a qué criterios utilizar para estimar el potencial de un mercado, la importancia relativa de cada criterio y cómo aplicarlos.

En cuanto a los planteamientos teóricos en relación a la SME existe una gran primera división que clasifica dichos planteamiento en cualitativos y cuantitativos. Mientras que los primeros han ido perdiendo terreno en función a sus limitaciones, las cada vez más sofisticadas técnicas estadísticas han generado un mayor interés y una mayor aceptación de los segundos.

En relación al nivel de análisis algunos autores realizan el análisis de SME en base a una macro segmentación mientras que otros realizan una micro segmentación, así como también se ubican investigaciones que recurren a una combinación de ambas metodologías. En general encontramos acuerdo en señalar las limitaciones de los enfoques que solo contemplan el análisis a nivel macro, en este sentido Douglas y 
Craig señalan; que el elemento clave es que dos países pueden tener contexto macro ambientales muy similares, pero pueden diferir radicalmente en su contextos a otros niveles (Douglas \& Craig, 2011).

Como tercer punto de este ensayo, presentamos las propuestas de adaptación de metodologías de SME con el fin de considerar las características de los mercados emergentes que hemos identificado.

En primer término, identificamos el Índice Integral de Oportunidades de Mercado (OMOI) presentado por Cavusgil (1997). El autor desarrolla una metodología con el fin de cuantificar y clasificar el potencial de mercado de una lista de países considerados emergentes. Desarrolla un índice estandarizado en base a trece variables que responden a los ámbitos económico, político y social. Estas variables son agrupadas en siete dimensiones con el objetivo de determinar el atractivo general de un mercado.

En ese sentido observamos que el análisis realizado está focalizado a nivel macro y que el resultado final de la aplicación de esta metodología es una categorización de países emergentes. El autor reconoce las limitaciones de esta herramienta circunscribiendo su utilidad a la etapa de evaluación y clasificación de estos países, recomendando un análisis más exhaustivo para avanzar en el proceso de SME.

Aunque el autor no lo menciona, su enfoque y metodología reconocen implícitamente que no resultaría adecuado realizar una evaluación de mercados emergentes junto con países desarrollados con el fin de seleccionar un mercado externo de destino. Un factor interesante de mencionar es que el autor actualiza su análisis todos los años.

Un segundo aporte es realizado por Arnold \& Quelch (1998), estos autores señalan ciertos factores por los que ellos consideran que el método tradicional de evaluación de mercados no es aplicable a los mercados emergentes. Un primer punto es la dependencia de data macroeconómica y poblacional que puede ser inexacta o estar desactualizada. El segundo punto también está relacionado con la disponibilidad de datos de operaciones como niveles de ventas o número de distribuidores. Finalmente el tercer reconoce que los modelos de SME utilizados son generalmente evaluaciones "instantáneas" estáticas en lugar de evaluaciones dinámicas, ignorando de esta manera el potencial a largo plazo que los mercados emergentes y su rápido ritmo de cambio.

Arnold \& Quelch (1998) presentan un "modelo anidado" para la evaluación de los ME. Se trata de un modelo secuencial que va de lo general a lo específico y por lo tanto haciendo uso de indicadores macro a nivel inicial para luego descender a un nivel micro buscando data correspondiente a la industria bajo estudio.

Los aportes de estos autores están relacionados con la evaluación del potencial de mercado a largo plazo utilizando datos y tendencias de población y PBI, obteniendo el potencial de mercado relacionando la población del país emergente con la diferencia entre la riqueza del mercado emergente y los mercados desarrollados. Una contribución importante de estos autores es el concepto de "conversión de beneficio potencial". Éste concepto se refiere a la capacidad de las empresas de "extraer valor del mercado", indicando que ello dependerá del riesgo político y económico y del desarrollo de condiciones adecuadas en el sector comercial, como por ejemplo la infraestructura en transporte, distribución y telecomunicaciones. 
En este sentido podemos concluir que los autores reconocen las limitaciones de los enfoques de SME que descansan en un nivel macro, que aportan una herramienta para la evaluación del potencial del mercado a largo plazo, e inciden en la importancia de la infraestructura en el análisis.

Finalmente Sakarya, Eckman y Hyllegard (2007), al igual que los autores anteriores, estos autores reconocen las limitaciones de los enfoques tradicionales al depender los mismos de criterios macroeconómicos sin tener en cuenta el dinamismo y el potencial futuro de un mercado emergente. Presentan un modelo ecléctico, que recoge aportes de estudios anteriores, integrando en base a ello criterios culturales además de los económicos y realizando un análisis escalonado, que parte con la evaluación de variables macro para luego pasar a un análisis micro y un análisis industrial. Finalmente los autores realizan un estudio empírico con el fin de aplicar el modelo planteado a una industria específica; para ello evalúan las oportunidades de la industria de textiles y confecciones de Estados Unidos en Turquía.

\section{Conclusiones}

Si bien no existe consenso en la definición de mercados o países emergentes sí podemos concluir que existe consenso respecto a la importancia de los mismos hacia el futuro. Desde esta perspectiva resaltan tres características de los mercados emergentes que los diferencian de los mercados desarrollados; su inestabilidad la misma que afecta su predictibilidad (riesgo), su heterogeneidad, característica que puede generar mayor complicación en sus evaluaciones y su potencial de crecimiento (oportunidad).

Encontramos contribuciones en relación al tema de SME tanto desde la perspectiva de los negocios internacionales; por la importancia que la teoría le concede a la selección de mercados externos como factor clave para el futuro éxito en el proceso de internacionalización de las empresas; así como también desde la perspectiva del marketing internacional al reconocer que el marketing es contexto-dependiente.

La metodología que elija una empresa para realizar la selección de mercados externos debe ser sistemática y gradual; y debería permitir la adaptación de la misma en función a las características de la empresa.

En cuanto a las metodologías; los métodos cuantitativos presentan tres ventajas principales; (1) reducen la subjetividad, (2) permiten que las empresas consideren mercados ubicados más allá de sus vecinos, y (3) Permiten evaluar un gran número de mercados, como es subrayado por los autores Papadopoulos y Denis.

La discusión en torno a la selección de mercados externos debe considerar tanto la metodología de filtración, como también el uso de indicadores y ponderaciones.

En cuanto al primer punto los teóricos aceptan haber llegado a un acuerdo al respecto, presentándose metodologías que buscan el agrupamiento de países mientas que otras buscan la priorización de los mercados. En cuanto al nivel de análisis algunos modelos se desarrollan a un nivel macro mientras que otras profundizan a un nivel micro. 
Los aportes teóricos encontrados en relación a las metodologías de selección de mercados externos aplicadas a países emergentes sostienen que las variables utilizadas en la mayoría de estudios a nivel macro no tienen la capacidad de evaluar la capacidad de crecimiento de las economías emergentes.

En función a las características de los países emergentes es recomendable en el análisis para la SME llegar hasta el nivel micro e incluir dentro del set de variables a utilizar aquellas que evalúen su potencial de crecimiento y la continuidad de sus reformas socio económicas lo que está directamente relacionado con un punto crucial; que estos países continúen mejorando sus marcos institucionales.

El esfuerzo debe ir por la recomendación de fuentes de información, variables que no estén correlacionadas para las cuales se cuente con datos y estadísticas disponibles para su utilización que sean capaces de recoger las características particulares de los mercados emergentes.

Concluimos que la metodología de selección de mercados externos fue desarrollada considerando condiciones y parámetros que se registraban en los países desarrollados. La estabilidad de estos mercados permitía establecer la predictibilidad de los mismos, situación que no se repite en los mercados emergentes. Esta inestabilidad este establece tanto oportunidades como riesgos, que deben ser considerados para una adecuada selección de mercados externos.

Los aportes encontrados tienden a reforzar la idea de que si bien no se invalida la metodología de selección de mercados externos, la realidad de los mercados emergentes estaría estableciendo limitantes a la misma.

Al plantearnos la siguiente interrogante, ¿Cómo lograr que la evaluación de los mercados emergentes como posibles mercados de destino para los negocios internacionales tome en cuenta la inestabilidad inherente al proceso de formación de su institucionalidad?, surge la oportunidad de aportar a esta línea de investigación, a través del análisis de las economías emergentes a la luz de la teoría institucional y la teoría industrial con el fin de encontrar a través de este análisis un apoyo teórico a los cambios que sean sugeridos a la SME aplicada a los ME.

Así también resultaría adecuado pensar en contribuir a esta línea de investigación realizando estudios empíricos que permitan evaluar los nuevos aportes que buscan mejorar el modelo de selección de mercados externos en diferentes realidades.

\section{Referencias bibliográficas}

Arnold, D. J., \& Quelch, J. A. (1998). New Strategies in Emerging Markets. Sloan Mangement Review, 7-20.

Berbel Pineda, J. M., Ramón Jerónimo, M. A., \& Vásquez Carrasco, R. (2012). La Selección de Mercados Preferentes Como Clave en la Internacionalización Empresarial. TEC Empresarial, 21 - 33. 
Brouthers, L. E., \& Nakos, G. (2005). The Role of Systematic International Market Selection on Small Firms' Export Performance. Journal of Small Business Management 43 (4), 363-381.

Cavusgil, S. T. (1985). Guidelines For Export Market Research. Business Horizons, 2733.

Cavusgil, S. T. (1997). Measuring The Potencial Of Emerging Markets: An Indexing Approach. Business Horizons, January - February, 87- 91.

Cavusgil, S. T., Knight, G., Riesenberger, J., \& Yaprak, A. (2009). Conducting Market Research For International Business. New York: Business Expert Press, LLC.

Cellich, C. (1998). The Big Ten: The Big Ten Emerging Markets and How They Will Change Our Lives by Jeffrey E. Garten. Journal of International Marketing, Vol. 6, № 4, 94-97.

Cronin, P., Ryan F., \& Coughlan M. (2008). Undertaking a Literature Review: A Step-byStep Approach. British Journal of Nursing, Vol. 17, N¹, 38-43.

Douglas, S. P., \& Craig, C. S. (2011). The Role of Context in Assessing International Market Opportunities. International Marketing Review, Vol 28 N² 2, 150-162.

Evirgen, C., Bodur, M., \& Cavusgil, S. T. (1993). Information Needs of Exporters: An Empirical Study of Turkish Exporters. Marketing Intelligence \& Planning, 28-36.

Ghemawat, P. (2004). Distance Still Matters, The Hard Reality of Global Expansion. Harvard Business Review, 1-12.

Green, R. T., \& Allaway, A. W. (1985). Identification of Export Opportunities: A ShiftShare Approach. Journal of Marketing, Vol. 49, N 1, 83-88.

Hernández, R., Fernández, C., \& Baptista, M. (2010). Metodología de la Investigación. México, D.F.: McGRAW-HILL / INTERAMERICANA EDITORES, S.A. DE C.V.

Jansson, H. (2007). International Business Strategy in Emerging Country Markets. Glos, UK: Edward Elgar Publishing, Inc.

Johanson, J., \& Vahlne, J.-E. (1977). The Internationalization Process of the Firm A Model of Knowledge Development and Increasing Foreign Market Commitments. Journal of International Business Studies, Vol. 8, № 1 (Spring - Summer), 23-32.

Johanson, J., \& Vahlne, J.-E. (2009). The Uppsala Internationalization Process Model Revisited: From Liability of Foreignness to Liability of Outsidership. Journal of International Business Studies, Vol. 40, N 9, 1411-1431.

Khanna, T., \& Palepu, K. (1997 (July - August)). Why Focused Strategies May Be Wrong For Emerging Markets. Harvard Business Review, 41-51.

Khanna, T., \& Palepu, K. G. (2010). Winning in Emerging Marekts: A Road Map for Strategy and Execution. Boston: Harvard Business Press. 
Khanna, T., Palepu, K. G., \& Sinha, J. (2005 (June)). Stategies That Fit Emerging Markets. Harvard Business Review, 63-76.

Kumar, V., Stam, A., \& Joachimsthaler, E. A. (1994). An interactive Multicriteria Approach To Identifying Potential Foreign Markets. Journal of International Marketing, Vol. 2, N¹, 29-52.

Magnusson, P., Westjhon, S. A., Gordon, G. L., \& Aurand, T. W. (2012). Environmental Dynamics and First- Movers Advantages in Emerging Markets. The Marketing Management Journal, 17-34.

Melin, L. (1992). Internationalization as a Strategy Process. Strategic Management Journal, Vol.13, Special Issue: Fundamental Themes in Strategy Process Research, 99118.

Morosini, P., Shane, S. y Singh, H. (1998), "National Culture Distance and Cross-Border Acquisition Performance”, Journal of International Business Studies, Vol. 29, pp. 13758.

Papadopoulos, N., \& Denis, J.-E. (1988). Inventory, Taxonomy and Assessment of Methods For International Market Selection. International Marketing Review, 38-51.

Papadopoulos, N., \& Martín, O. M. (2011). International Market Selection and Segmentation: Perspectives and Challenges. International Marketing Review, Vol. $28 \mathrm{~N}^{\circ}$ 2, 132-149.

Papadopoulos, N., Chen, H., \& Thomas, D. R. (2002). Toward a Tradeoff Model for international Market Selection. International Business Review 11, 165-192.

Pels, J., \& Kidd, T. (2012). Characterizing Emerging Markets. Organizations and Markets in Emerging Economies, Vol. 3, $\mathrm{N}^{\circ} 2(6), 8-22$.

Peng, M. W. (2009). Global Business. Mason, OH: South-western Cengage Learning.

Porter, M.E. (1990), The Competitive Advantage of Nations, The Free Press, New York, NY.

Rahman, S. (2000). Toward Developing an International Market Selection Decision Framework. ANZMAC, 1029-1033.

Rahman, S. H. (2000). Organisational and Strategic Considerations in International Market Selection. International Journal of Applied International Business, 1-14.

Rahman, S. H. (2006). International Market Selection Process: An Investigation Of The Relevance Of Business Operating Environment. Journal of International Business Research, Volume 5, Number 1, 73-86.

Russow, L. C., \& Okoroafo, S. C. (1996). On The Way Towards Developing a Global Screening Model. International Marketing Review, Vol. 13 N¹, 46-64. 
Sakarya, S., Eckman, M., \& Hyllegard, K. H. (2007). Market Selection For International Expansion. Assessing Opportunities in Emerging Markets. International Marketing Review Vol $24 \mathrm{~N}^{\circ} 2$, 208-238.

Sheth, J. N. (2011). Impact of Emerging Markets on Marketing: Rethinking Existing Perspectives and Practices. Journal of Marketing, Vol. 75 (July), 166-182.

Steenkamp, E., Rossow, R., Viviers, W., \& Cuyvers, L. (2009). Export Market Selection Methods and the Identification of Realistic Export Opportunities for South Africa Using a Decision Support Model. Pretoria: TIPS.

Sunje, A., \& Civi, E. (2000). Emerging Markets: A Review Of Conceptual Frameworks. First International Joint Symposium on Business Administration: Challenges for Business Administators in the New Millennium, (pages. 203-216). Canakkale.

Verbeke, A. (2009). International Business Strategy. Cambridge, UK: Cambridge University Press.

Wood, V. R., \& Robertson, K. R. (2000). Evaluating International Markets: The Importance of Information by Industry, by Country of Destination and by Type of Export Transaction. International Marketing Review, 34-55.

World Trade Organization. (2013). World Trade Report 2013. Factors Shaping The Future of World Trade. Ginebra: World Trade Organization. 